\section{Is Pakistan doing Enough to Eradicate Rabies by 2030?}

Sir,

Unfortunately, rabies is considered poor men's disease in Pakistan, despite the fact that it leads to 2,000-5,000 deaths annually. ${ }^{1}$ Recently, an upward trend has been noted, but disease burden remains grossly underestimated as cases go unreported. ${ }^{1}$

Lack of awareness in the community remains one of the biggest challenges faced by the country in its fight against rabies. A majority of victims do not take dog bites seriously and a delay in seeking post-exposure prophylaxis (PEP) results in increased mortality. ${ }^{1}$ Till 2015, the nerve tissue vaccine was being used as PEP all over Pakistan, ${ }^{2}$ which had devastating side effects and led to an increase in anti-vaccination sentiment in the population. ${ }^{3,4}$

It is extremely necessary to establish a comprehensive rabies education programme, which increases awareness amongst the Pakistani population, particularly low socioeconomic communities. It is imperative to educate the masses regarding the fatality of the disease and importance of PEP. ${ }^{5}$ Pre-exposure prophylaxis should be emphasised amongst the high risk population, such as laboratory staff workers, animal handlers and inhabitants of rural areas, who indulge in outdoor activities. Seeking early medical intervention should be promoted along with encouraging proper wound care and washing, followed by vaccine administration. The use of home remedies such as chili powder and oils should be strictly discouraged. ${ }^{5}$

Pakistan was previously importing anti-rabies vaccine (ARV) from India, but due to increasing tension between the two countries, we are facing an acute shortage of ARV since 2019. ${ }^{4}$ The National Institute of Health $(\mathrm{NIH})$ has been unable to produce enough ARV to meet local demands. ${ }^{4}$ Currently, most rabies centres in Pakistan use the five-dose IM regime. ${ }^{6}$ It is necessary to train healthcare workers to administer vaccines intradermally to increase cost-effectiveness and patient compliance.

Since dogs are considered inessential for economic growth, their existence is ignored and canine population control is neglected. ${ }^{1}$ Mass culling is still practised; but fails, as it does not provide an effective long-term solution to the problem. WHO recommendations suggest mass dog vaccination and neutering to establish herd immunity. ${ }^{5}$ Some South-East Asian countries have successfully reported a decline in canine rabies by following this a pproach. ${ }^{5}$

In order to meet the goal of rabies eradication by 2030 , prompt action needs to be taken at all levels of government, health departments and community. Using the one health model, ${ }^{5} \mathrm{a}$ comprehensive national programme is required; one that will be responsible for creating a registry for collecting, reporting and sharing of data. It should actively support sampling of infected animals and seek funding for the cause. It would also be responsible for training dog catchers and vaccinators. It is only with unanimous effort, inter-sectorial and inter-regional collaboration that we may hope to meet our goal of a rabies-free Pakistan by 2030.

\section{CONFLICT OF INTEREST:}

The authors declared no conflict of interest.

\section{AUTHORS' CONTRIBUTION:}

AM, SAH: Conceived the idea and contributed to the literature search, writing, editing, final review, and submission of this commentary equally.

\section{REFERENCES}

1. Can Pakistan meet the global goal of rabies eradication by 2030? Possible approaches. Uvas.edu.pk. 2020 [cited 5 April 2020].

2. Salahuddin N, Gohar MA, Baig-Ansari N. Reducing cost of rabies post-exposure prophylaxis: experience of a tertiary care hospital in Pakistan. Plos Neglected Tropical Diseases 2016; 10(2). doi: 10.1371/journal.pntd.0004448.

3. Rupprecht CE, Salahuddin N. Current status of human rabies prevention: Remaining barriers to global biologics accessibility and disease elimination. Expert Rev Vaccines 2019; 18(6):629-40. doi: 10.1080/14760584.2019. 1627205.

4. Salahuddin N. Vaccine conundrum. Dawn.Com. 2020 [cited 5 April 2020].

5. World Health Organization. WHO expert consultation on rabies: Third report. World Health Organisation; 2018 Aug 31.

6. Kessels J, Tarantola A, Salahuddin N, Blumberg L, Knopf L. Rabies post-exposure prophylaxis: A systematic review on abridged vaccination schedules and the effect of changing administration routes during a single course. Vaccine 2019; 37:A107-17. doi: 10.1016/j.vaccine.2019.01.041.

Ayesha Mubashir and Syeda Asfia Hussain

Department of Internal Medicine, Dow University of Health Sciences, Karachi, Pakistan

Correspondence to: Dr. Ayesha Mubashir, Department of Internal Medicine, Dow University of Health Sciences, Karachi, Pakistan

E-mail: ayesha.mubashir12@yahoo.com

Received: May 29, 2020; Revised: November 08, 2020; Accepted: November 29, 2020

DOI: https://doi.org/10.29271/jcpsp.2021.05.614 